\title{
Use of Poly-Lactic Acid (PLA) to Enhance Properties of Paper Based on Recycled Pulp
}

\author{
Klaus Doelle, Anh T. Le, Thomas E. Amidon, Biljana M. Bujanovic \\ Department of Paper and Bioprocess Engineering, State University of New York College of Environmental \\ Science and Forestry, Syracuse, USA \\ Email: kdoelle@esf.edu
}

Received 6 June 2014; revised 26 June 2014; accepted 2 July 2014

Copyright (C) 2014 by authors and Scientific Research Publishing Inc.

This work is licensed under the Creative Commons Attribution International License (CC BY). http://creativecommons.org/licenses/by/4.0/

(c) (i) Open Access

\begin{abstract}
Nowadays, recycled paper is broadly used due to environmental reasons. Furthermore, the addition of starch as a dry strength additive improves the properties of recycled paper. Poly-Lactic Acid (PLA), a product from bio-refinery process, has recently been shown to act as a promising strength additive that could be used in combination with starch to further improve the strength of paper. In this study, the use of PLA of three molecular weights (MW) in combination with four different starches was investigated. Three recycled pulps from different origins, with the kappa number of 27.9 to 66 were used. Paper handsheets were made, and selected paper properties were tested. The results indicate that handsheets properties were influenced by the MW of PLAs, the type of starch used, and the lignin content of the pulp. The paper handsheets made from lignin-rich pulp (pulp A, kappa number 66), combined with 0.1\% medium MW PLA (PLA_1) and 0.9\% cationic starch containing $0.43 \% \mathrm{~N}$ gave the highest improvement for tensile strength, wet tensile strength, air and water resistance. This result verifies that a higher kappa number pulp has better attraction to the hydrophobic PLA. Moreover, the higher charge cationic starch led to higher tensile strength due to the increase of affinity to the anionic fiber surface. Interestingly, results show that amphoteric starch is a promising substitute for high cationic charge starch when combined with the medium MW PLA to improve tensile strength of paper. This study demonstrated that a starch-PLA blend represents a promising approach in improving properties of recycled paper.
\end{abstract}

\section{Keywords}

Poly-Lactic Acid (PLA), Recycled Pulp (OCC), Starch, Paper Properties, Paper Handsheets

\section{Introduction}

Currently, papermakers are focusing on how to improve strength for paper. This can be measured by tensile

How to cite this paper: Doelle, K., Le, A.T., Amidon, T.E. and Bujanovic, B.M. (2014) Use of Poly-Lactic Acid (PLA) to Enhance Properties of Paper Based on Recycled Pulp. Advances in Chemical Engineering and Science, 4, 347-360.

http://dx.doi.org/10.4236/aces.2014.43038 
strength, bursting strength, or internal bonding strength. There are various ways to increase the strength of paper such as using refining, wet pressure, or additives. However, use of mechanical actions like refining could bring about the reduction in opacity, brightness, dimensional stability or porosity [1]. Therefore, use of additives is very common and has been employed in most paper mills. Starch is used most frequently today as a dry strength additive in the paper industry. Starches can significantly improve the mechanical properties of paper such as tensile strength. Also, starches are used as retention aid, surface sizing agent, coating binder, and adhesive in corrugated board and other converting operations [2].

Beside the excellent contributions to the strength and other properties of paper, the use of starches can lead to several difficulties for papermaking process. One of the current major problems when using starches is the loss of starch to the white water system. Starches are water soluble, hydrophilic polymers [3]. When the amount of starch added to the system is high, the retention of starch on the paper web becomes lower and then starch would be lost to the white water system due to its hydrophilic properties. The loss of starches results in increasing production costs, and in the long term of operation, it can cause microbiological problems that negatively affect the runnability of machines as well as the quality of paper. Moreover, because starch is mainly considered as a dry wet strength agent, it is not known to be a contributor to the wet strength, air and water resistance of paper. Therefore, it is necessary to study new additives to use together with starch to further increase the strength of paper, reduce the consumption of starch, reduce the microbiological problems, and improve wet strength, air and water resistance of paper.

In an attempt to fulfil these needs, poly-lactic acid (PLA) had been introduced to the wet end system. PLA has been found to be a potential alternative for starch, and it is also a biodegradable product. Furthermore, PLA can be produced from natural resources like corn, but also woody biomass. Recently PLA has been shown in the laboratory study to have the function of a strength additive [4] [5]. The laboratory result was reported that the blend of PLA with cationic starch as a wet end additive improved the tensile strength of paper. Regarding to Gong, several virgin pulps had been used including Norway spruce thermo-mechanical pulp (TMP), hardwood unbleached kraft pulp, and softwood bleached kraft pulp. Two cationic starches that have different nitrogen contents, and PLA with molecule weight of 20,000 - 30,000 were employed.

In this research, three types of recycled pulp were used instead of the virgin pulps. This was done because recycled paper has been used broadly currently due to environmental impact. Furthermore, it has been shown that some paper properties from recycled pulp could be comparable to that of virgin pulp. For example, after several times of recycling, mechanical pulp still gives better density, better burst strength and better tensile strength compared to the never-dried mechanical pulp [6]. However, for chemical pulp, important paper properties like burst and tensile strength decrease as the number of recycles increase. The reason for the decrease in strength is due to the loss of bonding potential between fibers after the fibers were dried in the paper drying process, commonly known as "hornification" phenomenon. One of the methods to recover the strength of paper is to use the additives such as modified starch.

In this present paper, we report the properties of paper handsheets made from the three recycled pulps, combined with three types of PLA and four different starches.

\section{Experimental}

\subsection{Materials}

Recycled pulp (Pulp A) was donated by a paper company in Central New York. Kappa number of this pulp was 66. The initial Canadian Standard Freeness (CSF) was $606 \mathrm{ml}$. The pulp was then refined to CSF of $305 \mathrm{ml}$ which is close to CSF of $300 \mathrm{ml}$. Regarding TAPPI (Technical Association of Pulp and Paper Industry) T 200, CSF of $300 \mathrm{ml}$ is recommended because many pulps show a maximum strength at this freeness. Recycled pulp (Pulp B) was supplied by a paper company from Vietnam. Kappa number was found at 27.9 and the initial CSF was found at $419 \mathrm{ml}$. The pulp was refined to a CSF of $286 \mathrm{ml}$. Recycled pulp (Pulp C) was the same as Pulp A but it was refined to a different CSF, $298 \mathrm{ml}$. Linerboard samples were obtained from a paper mill in Central New York. The linerboard was produced from 100\% recycled fibers, and was treated with the internal sizing agent alkyl ketene dimer (AKD).

Cationic starch 1 (CAT.N1) is a commercial product and was supplied by a company in the United States. The nitrogen content of this starch is $0.28 \%$. Cationic starch 2 (CAT.N2) with $0.43 \%$ nitrogen content, was supplied by a different chemical company. Amphoteric starch (AS), a commercial product, was provided by the 
same company as for the CAT.N1. The nitrogen and phosphate content were $0.28 \%$ and minimum $1 \%$, respectively. Anionic starch (ANS), a commercial product, was obtained from the same company as the CAT.N1.

There are three different types of Poly-lactic acid (PLA) employed. They were all purchased from Polysciences, Inc in the United States. Poly-lactic acid 1 (PLA_1) is poly (dl-lactic acid) with a molecular weight MW 20,000 - 30,000. Poly-lactic acid 2 (PLA_2) is poly (l-lactic acid) with a molecular weight MW 1600 2400. Poly-lactic acid 3 (PLA_3) is poly (l-lactic acid) with a molecular weight MW 140,000 - 160,000.

\subsection{Methods}

\subsubsection{TAPPI Methods}

Kappa number of the recycled pulp was measured in accordance with TAPPI T 236 om-06, "Kappa number of pulp”. The CSF was measured by T 227 om-09 "Freeness of pulp (Canadian standard method)”. Paper handsheets were made followed T 205 sp-06, "Forming handsheets for physical tests of pulp”. Paper specimens were prepared using the T220 sp-10, "Physical testing of pulp handsheets". Grammage and Thickness of handsheets were determined by the T220 sp-10. The tensile strength was performed following T 494 om-06, "Tensile properties of paper and paperboard (using constant rate of elongation apparatus)”. The wet tensile strength was measured on the same machine used for dry tensile strength, and the method used was T 456 om-10, "Tensile breaking strength of water-saturated paper and paperboard ("Wet tensile strength”). The Gurley porosity was performed by following the T 460 om-11, “Air resistance of paper (Gurley method)”. The tear strength was done by following the T 414 om-12, "Internal tearing resistance of paper (Elmendorf-type method)”. The Cobb values were obtained by the T441 om-09, "Water absorptiveness of sized (non-bibulous) paper, paperboard, and corrugated fiberboard (Cobb test)”. Water drop was measured by T 835 om-08, "Water absorption of corrugating medium: water drop absorption test”.

\subsubsection{PLA Treatment}

Because PLA is not soluble in water, it was dissolved in organic solvents. Furthermore, because different molecular weights of PLA were used, two different solvents were used. PLA_1 was dissolved in acetone easily under normal stirring condition and a short period of time. PLA_1 can be dissolved completely in a minimal amount of acetone. In this study, $0.02 \mathrm{~g}$ of PLA_1 was completely dissolved in $20 \mathrm{ml}$ acetone. PLA_2 was dissolved in acetone but under long and high stirring condition. PLA_3 was dissolved in p-dioxane under long and high stirring condition.

\subsubsection{Starch Preparation}

Starch in the form of powder was mixed with distilled water and then cooked at the consistency $0.1 \%$ at temperature $95^{\circ} \mathrm{C}-98^{\circ} \mathrm{C}$ for 30 minutes under constant stirring. During the cooking, a flask containing the starch solution was covered by a small aluminium tray to prevent the evaporation of water.

\subsubsection{Mixing of Starch-PLA}

After PLA is dissolved in acetone, it was mixed with the cooked starch and they together formed a clear and stable solution. Then the starch-PLA mixture was added to the pulp slurry and agitated for about 5 minutes to get an even mixture of pulp, starch and PLA.

\section{Results and Discussion}

Selected properties of paper handsheets made from the three different pulps, from the four different starches and from the three different PLAs are presented as following discussions. Table 1 is introduced to show the repeatability of different properties. This table will be used for comparison purpose in the following discussions.

\subsection{Effect of CAT.N1 on Paper Properties of Pulps A and B}

\subsubsection{Effect on Strength Properties}

Figure 1 shows the effect of this starch on the strength properties of paper made from the two different pulps, Pulp A and Pulp B.

For Tensile Index (TI), Figure 1 shows that for blank handsheet, the TI of paper made from Pulp A (33.10 
Table 1. Repeatability of test results.

\begin{tabular}{ccc}
\hline Tests & Repeatability & Recommended by TAPPI \\
\hline Tensile Strength (TS) & $5 \%$ & T494 \\
Wet Tensile Strength (WTS) & $4 \%$ & T456 \\
Tear & $4 \%$ & T414 \\
Gurley Porosity & $8 \%$ & $\mathrm{~T} 460$ \\
Water Drop & $13 \%$ & $\mathrm{~T} 835$ \\
Cobb & $2.5 \%$ & $\mathrm{~T} 441$ \\
\hline
\end{tabular}

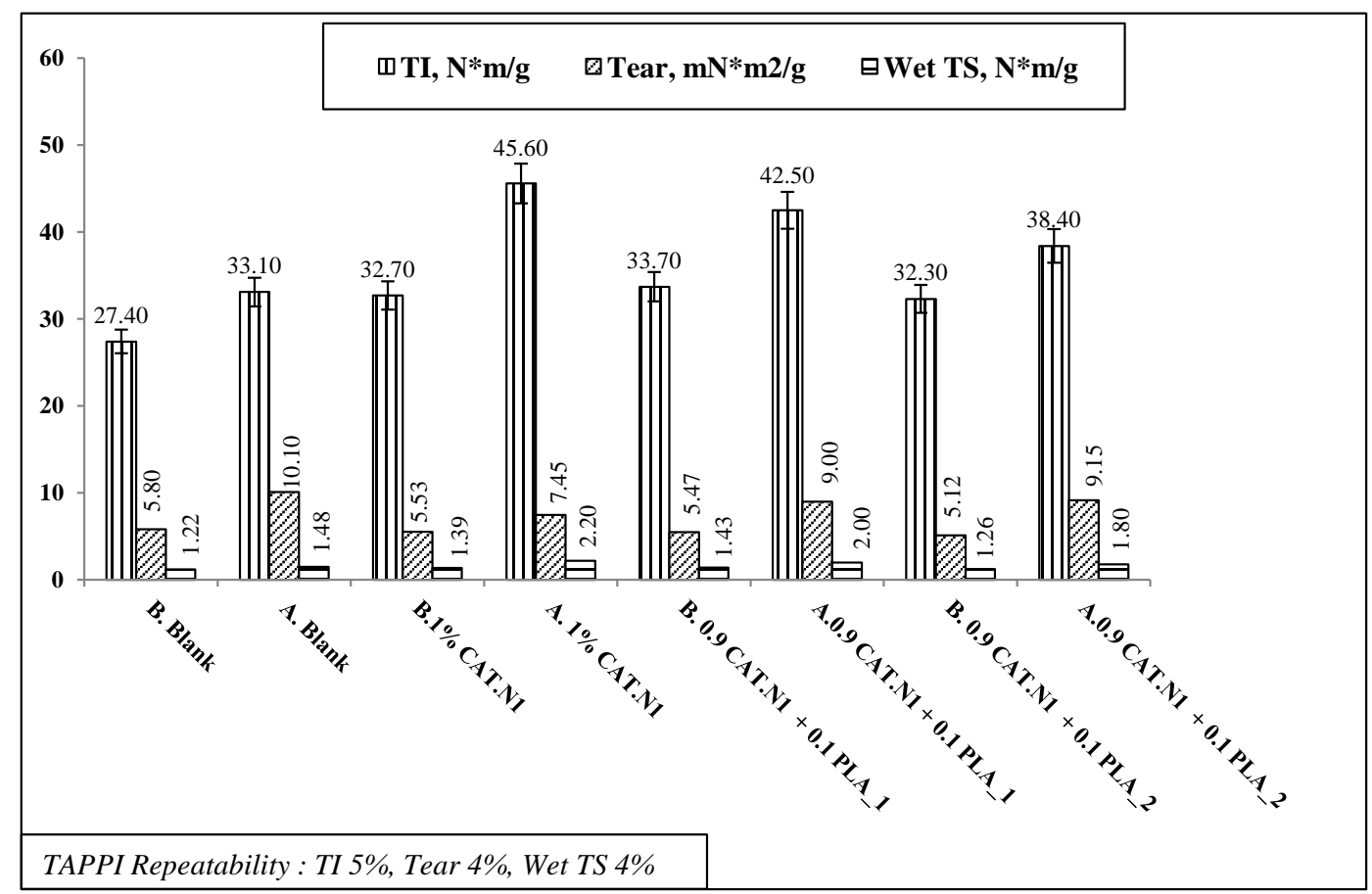

Figure 1. Effects of CAT.N1 on strength properties of Pulps A and B.

$\mathrm{Nm} / \mathrm{g})$ is greater than that of paper made from Pulp B $(27.40 \mathrm{Nm} / \mathrm{g})$. When $1 \%$ CAT.N1 is used, TI of paper made from Pulp A increases rapidly to $45.60 \mathrm{Nm} / \mathrm{g}$ ( 37.8\%), while the TI of paper made from Pulp B increases to $32.70 \mathrm{Nm} / \mathrm{g}(19.3 \%)$. When $0.1 \%$ PLA_1 is introduced, TI of paper made from Pulp A decreases to 42.50 $\mathrm{Nm} / \mathrm{g}$ while TI of paper made from Pulp B slightly increases to $33.70 \mathrm{Nm} / \mathrm{g}$. With the introduction of PLA_2, TI of both paper made from Pulp A and Pulp B become lower to 38.40 and 32.30, respectively. Therefore, the results propose that CAT.N1 has better effect on TI of Pulp A at 1\%. And CAT.N1 has better effect on TI of Pulp B at $0.9 \%$ combining with $0.1 \%$ PLA_1.

Similar to TI, with the use of $1 \%$ CAT.N1 alone paper made from pulp A shows higher wet TS than paper from Pulp B (2.20 vs. $1.39 \mathrm{Nm} / \mathrm{g}$ ). However, when combining with $0.1 \%$ PLA, wet TS of paper from Pulp B slightly increases from 1.39 to $1.43 \mathrm{Nm} / \mathrm{g}$ while it decreases from 2.20 to $2.00 \mathrm{Nm} / \mathrm{g}$ for paper made from Pulp A.

Tear values decrease for both Pulp A and Pulp B compared to the blank experiment in all cases. Interestingly, for Pulp A, the use of PLA_1 and PLA_2 with the CAT.N1 leads to the increase of the tear index compared to the use of 1\% CAT.N1 only. Particularly, tear index of Pulp A + 0.9 CAT.N1 + 0.1 PLA_1 and Pulp A + 0.9 CAT.N1 + 0.1 PLA_2 are 9.00 and $9.15 \mathrm{mN}^{*} \mathrm{~m}^{2} / \mathrm{g}$ respectively, which are higher than $7.45 \mathrm{mN}^{*} \mathrm{~m}^{2} / \mathrm{g}$ of the $1 \%$ CAT.N1. The results indicate that the combination of the medium charge cationic starch CAT.N1 with either PLA_1 or PLA_2 provides benefits for tear strength when the high kappa number pulp is used. 


\subsubsection{Effect on Air and Water Resistance Properties}

Figure 2 presents the effect of starch CAT.N1 to the air and water resistance properties of paper made from the two different pulps, Pulp A and Pulp B.

For Gurley porosity, it can be observed from Figure 2 that the highest value is recorded for Pulp A + $0.9 \%$ CAT.N1 + 0.1\% PLA_1, at 16.50 seconds. Similarly, Pulp B + 0.9\% CAT.N1 + 0.1\% PLA_1 also shows high value at 16.00 seconds which is close to the Pulp $A$. The lowest value of porosity at 7.74 seconds is seen for the combination of Pulp A with CAT.N1 and PLA_2. These results propose that the presence of PLA_1 helped to fill pores in the paper structure, leading to a lower rate of air passing through paper structure.

For water drop results, Pulp A + 0.9\% CAT.N1 + 0.1\% PLA_1 still shows the longest time at 224.00 seconds. This is very a significant increase compared to paper made with 1\% CAT.N1 and the blank. However, when CAT.N1 combines with PLA_2, the time for one drop of water passing paper thickness decreases rapidly to 138.00 seconds. Once again, the results suggest that with the presence of hydrophobic PLA_1 fiber surfaces are covered resulting in decreasing the rate of water penetrated through paper's thickness.

\subsection{Effect of Starch CAT.N2 on Paper Properties of Pulps A and B}

\subsubsection{Effect on Strength Properties}

Figure 3 presents the effects of CAT.N2 on strength properties of paper made from Pulp A and Pulp B.

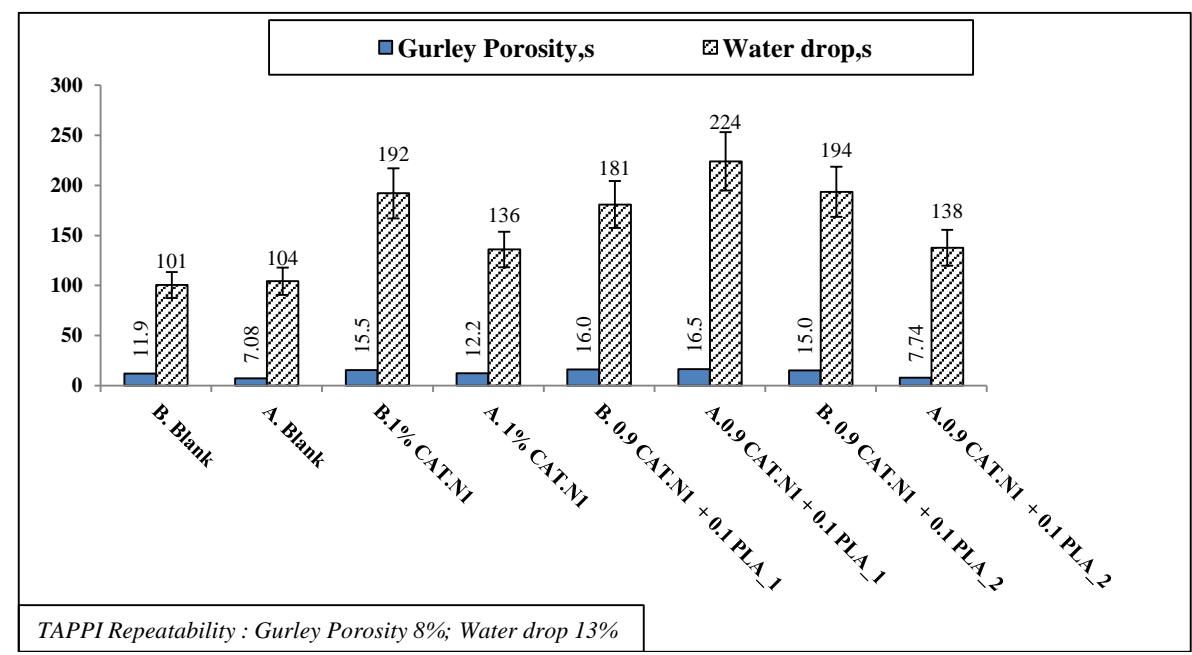

Figure 2. Effects of CAT.N1 on porosity and water drop properties of Pulps A and B.

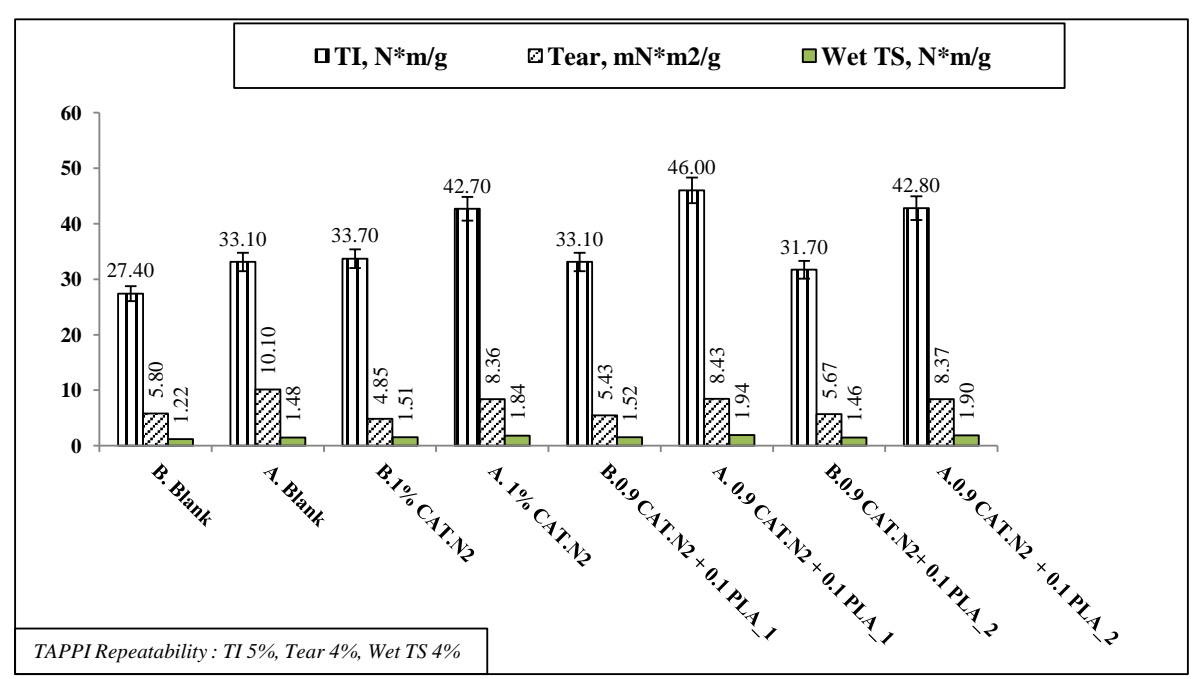

Figure 3. Effects of CAT.N2 on strength properties of Pulps A and B. 
With the use of 1\% CAT.N2, Pulp A shows the higher increase in TI than Pulp B. When 0.1\% PLA_1 is introduced, TI increases from 42.70 to $46.00 \mathrm{Nm} / \mathrm{g}$ for Pulp A but slightly decreases from 33.70 to $33.10 \mathrm{Nm} / \mathrm{g}$ for Pulp B. However, with the introduction of PLA_2, TI decreases for both paper made from Pulp A and Pulp B. Similarly, wet TS also appear to have the highest value at the combination of Pulp A, CAT.N2 with PLA_1 gives highest TI value.

Unlike the TI and wet TS, with the addition of CAT.N2 and/or PLA_1, PLA_2 tear indices go down for all the cases, including Pulp A and Pulp B compared to the tear of blank handsheet. However, interestingly, for Pulp B, tear strength increases when PLA_1 and PLA_2 are used, compared to the 1\% CAT.N2 only. Particularly, tear strength for Pulp A + 0.9 CAT.N2 + 0.1 PLA_1, and Pulp A + 0.9 CAT.N2 + 0.1 PLA_2 are 5.43 and $5.67 \mathrm{mN}^{*} \mathrm{~m}^{2} / \mathrm{g}$, higher than $4.85 \mathrm{mN}^{*} \mathrm{~m}^{2} / \mathrm{g}$ of the blank. The increase in tear strength is the result of the loss of interfiber bonding strength.

\subsubsection{Effect on Air and Water Resistance Properties}

Figure 4 presents the effect of CAT.N2 on Gurley porosity and water drop values of paper made from Pulp A and Pulp B.

CAT.N2 gives best response to water resistance ability of Pulp B, in the case of 1\% CAT.N2 (231.00 seconds) and $0.9 \%$ CAT.N2 + 0.1\% PLA_1 (229.00 seconds). Pulp A also shows a rapid increase from 127.00 to 172.10 seconds in water drop value with the use of 0.1\% PLA_1. In contrast, the use of PLA_2 with CAT.N2 decreases water drop value for both pulps.

It can also be seen that the highest value of Gurley porosity is shown for the combination of Pulp B $+0.9 \%$ CAT.N2 + 0.1\% PLA_1. That means the use of pulp with PLA_1 lowers the rate of air passing through the paper structure.

\subsection{Effects of AS on Paper Properties of Pulps A and B}

\subsubsection{Effect on Strength Properties}

Figure 5 shows the effect of AS on strength properties of paper made from Pulp A and Pulp B.

At 1\% AS, pulp A has better response in TI than Pulp B since the increase for Pulp A is 20\% (from 33.10 to $39.70 \mathrm{Nm} / \mathrm{g}$ ), while the increase for Pulp B is $\sim 9.5 \%$ (27.40 to $30.00 \mathrm{Nm} / \mathrm{g}$ ). When PLA_1 is added, the combination of PLA_1 with AS gives better response to Pulp A than Pulp B. That is because TI increases for Pulp A (to $44.90 \mathrm{Nm} / \mathrm{g})$ but almost keeps unchanged for Pulp B $(30.80 \mathrm{Nm} / \mathrm{g})$. Wet TS also give better response for Pulp A than Pulp B with the use of 1\% AS and 0.9\% AS + 0.1\% PLA_1. Tear values get lower than the blank handsheet when AS and PLA are used. That is the result of the increasing fibers bonding strength with the presence of starch and PLA.

\subsubsection{Effect on Air and Water Resistance Properties}

Figure 6 presents the effect of AS on porosity and water drop values of paper handsheets made from Pulp A and Pulp B.

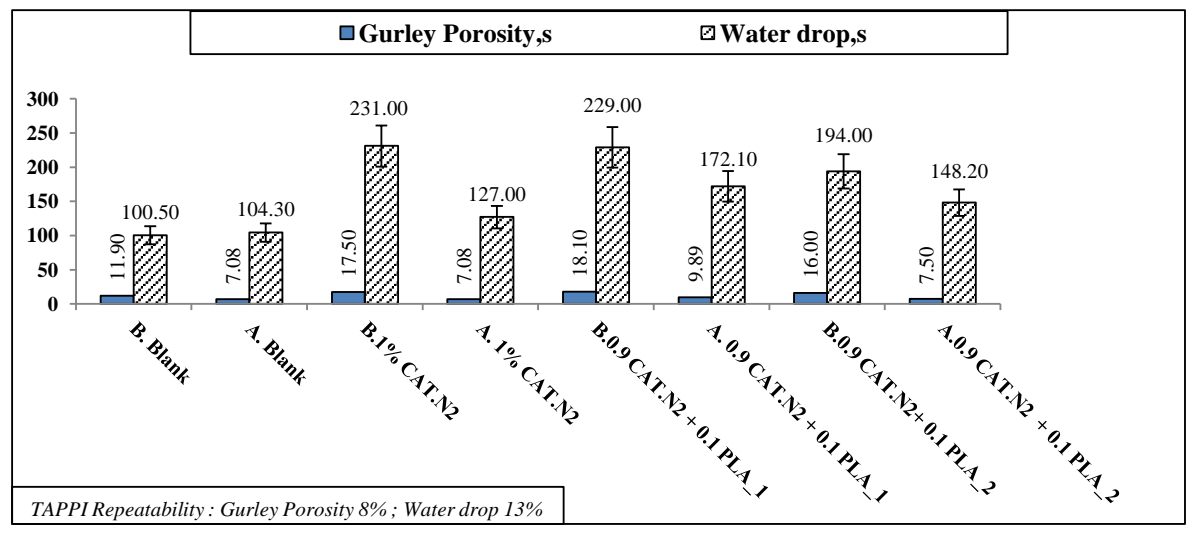

Figure 4. Effects of CAT.N2 on porosity and water drop of Pulps A and B. 


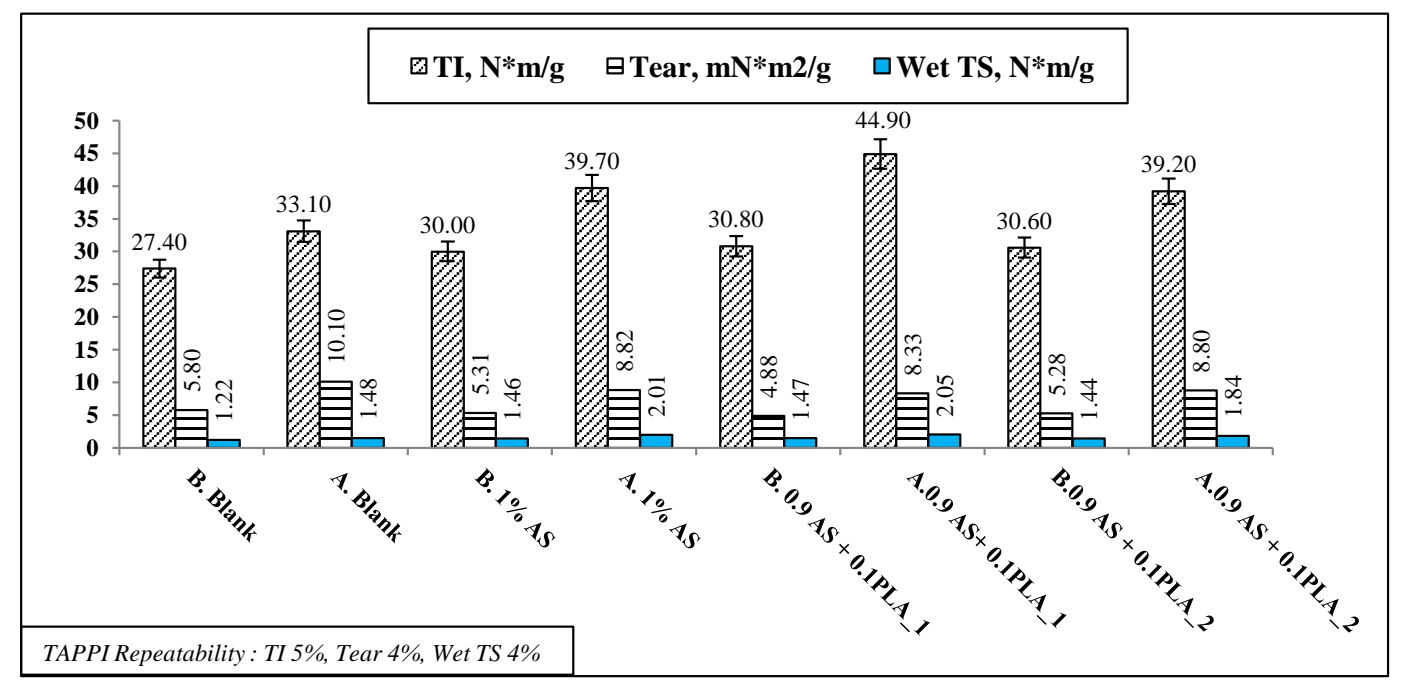

Figure 5. Effects of AS on strength properties of Pulps A and B.

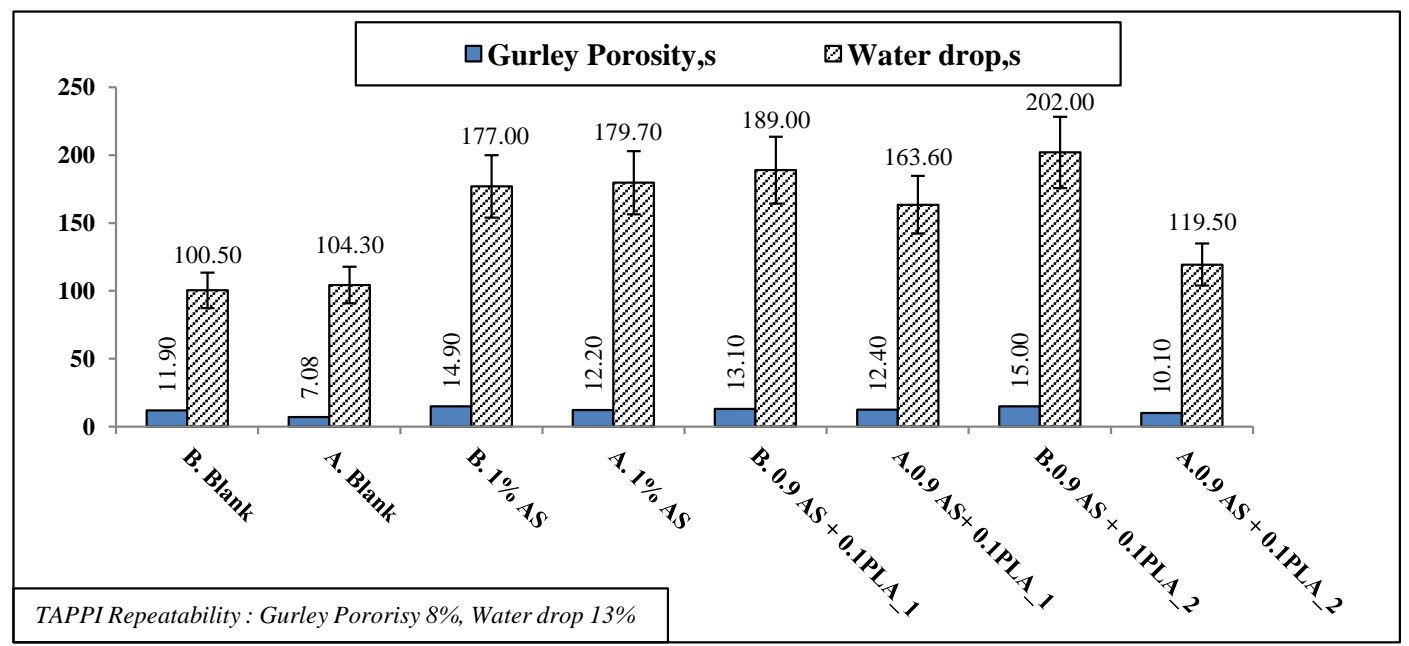

Figure 6. Effect of AS on porosity and water drop of Pulps A and B.

It is clear that at $1 \%$ AS water drop value is almost the same at 177.00 and 179.70 seconds for both paper made from Pulp A and Pulp B. When PLA_1 and PLA_2 are introduced at $0.1 \%$, paper handsheets from Pulp B have a better response than Pulp A. Particularly, the water drop values increase to 189.00 (PLA_1) and to 202.00 (PLA_2) seconds for Pulp B, but decrease to 163.60 (PLA_1) and 119.50 seconds (PLA_2) for Pulp A.

The $1 \%$ Gurley porosity also shows higher values for both paper made from Pulp A and Pulp B compared to that of the blank handsheets. The use of PLA_1combining with AS gives a better response for Pulp A, which slightly increases from 12.20 to 12.40 seconds, than for Pulp B which decreases from 14.90 to 13.10 seconds. In contrast to the use of PLA_1, the employment of PLA_2 has the opposite changes. That means Gurley porosity of paper from Pulp B is higher than that of Pulp A.

\subsection{Effects of Different Starches on Paper Properties of Pulp A}

\subsubsection{Effect on TI, Tear, Wet TS}

Figure 7 shows the effect of different starches (CAT.N1, CAT.N2, AS, ANS) on TI, tear, and wet tensile strength (wet TS) of paper handsheets made from Pulp A.

For the TI, in terms of paper handsheet made with $1 \%$ starch, Figure 7 shows that paper handsheets made with CAT.N1 at $1 \%$ has highest value of TI at $45.60 \mathrm{Nm} / \mathrm{g}$. The lowest value of TI is recorded for paper hand- 


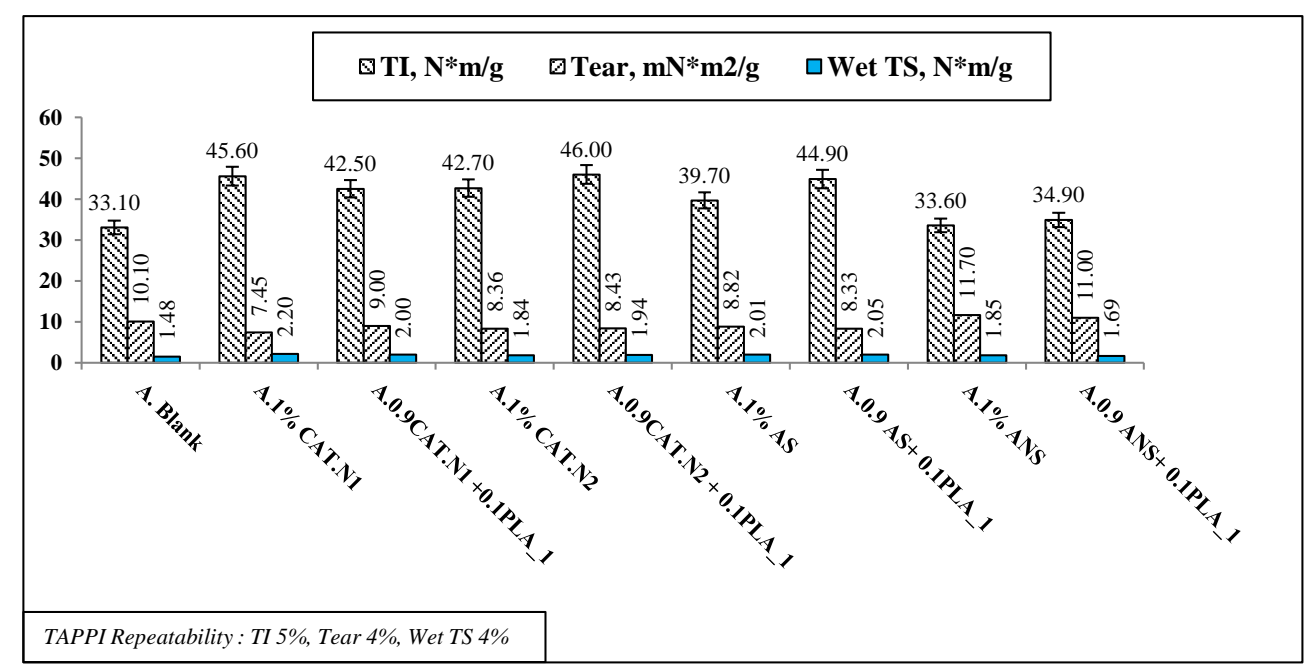

Figure 7. Effects of different starches on strength properties of Pulp A.

sheet made with 1\% ANS (anionic starch). The reason for the different effect is that cationic starch CAT.N1 contains cationic group which increases retention of starch on fibers. That leads to the better bonding degree and hence increasing TI. On the other hand, retention of anionic starch on fibers is less than that of cationic starch. Hence, the fibers bonding degree of paper made from anionic starch and the consequent TI is low.

Interestingly when combining with $0.1 \%$ PLA_1, the TI of paper made with $0.9 \%$ CAT.N1 becomes lower than TI of paper made with 1\% CAT.N1. In contrast, when combining with 0.1\% PLA_1, it can be seen that TIs of paper made with $0.9 \%$ CAT.N2 and with $0.9 \%$ AS are higher than that of paper made with 1\% CAT.N2 and 1\% AS, respectively.

For wet TS, the trend is very similar to TIs. It also shows that without PLA_1, paper made with 1\% CAT.N1 has highest value of wet TS. However, when combining with PLA_1, wet TS of paper made with CAT.N2 shows the best response.

For tear, for paper made with $1 \%$ starch alone the highest tear index is recorded for anionic starch $1 \%$ ANS at 11.70 $\mathrm{mN}^{*} \mathrm{~m}^{2} / \mathrm{g}$ and the lowest tear index $7.45 \mathrm{mN}^{*} \mathrm{~m}^{2} / \mathrm{g}$ is seen for paper made with $1 \%$ CAT.N1. That could be explained that when bonding between fibers increases, fibers become less flexible or more stiff and easy to be torn. When combining with $0.1 \%$ PLA_1, the highest tear index is still recorded for the anionic starch ANS at $11.00 \mathrm{mN}^{*} \mathrm{~m}^{2} / \mathrm{g}$.

\subsubsection{Effect on Air and Water Resistance}

Figure 8 shows the effect of four different starches (CAT.N1, CAT.N2, AS, ANS) on air and water resistance of paper handsheets made from Pulp A.

In terms of air resistance, when comparing the four types of starch alone at $1 \%$, both paper handsheets made with amphoteric starch AS and cationic starch CAT.N1 show the longest time at 12.20 seconds for air passing through paper. The shortest time (7.08 seconds) for air to pass through the paper sample is recorded for paper made with CAT.N2.

When combining with 0.1\% PLA_1, all the starch-PLA paper handsheets show the increase in time to let air pass through the paper. And the longest time is still recorded for paper made with CAT.N1 $(0.9 \%+0.1 \%$ PLA) at 16.50 seconds. That suggests that with the use of CAT.N1, more pores are filled in the paper structure.

For the water drop result, it can be seen clearly that when combining with $0.1 \%$ of PLA_1, cationic starch CAT.N1 also shows the longest time (224.00 seconds) to let one drop of water completely penetrate through the thickness of the paper sample. This suggests that with the present of hydrophobic characteristic of PLA, PLA helps to cover fibers and then repel water or reduce the rate of water penetration into paper.

\subsection{Effects of Different Starch Contents and Starch-PLAs on TI (Pulp A. CAT.N2)}

Figure 9 shows the TI of paper handsheets made from different starches ratios, and from different combination of PLAs with starches. 


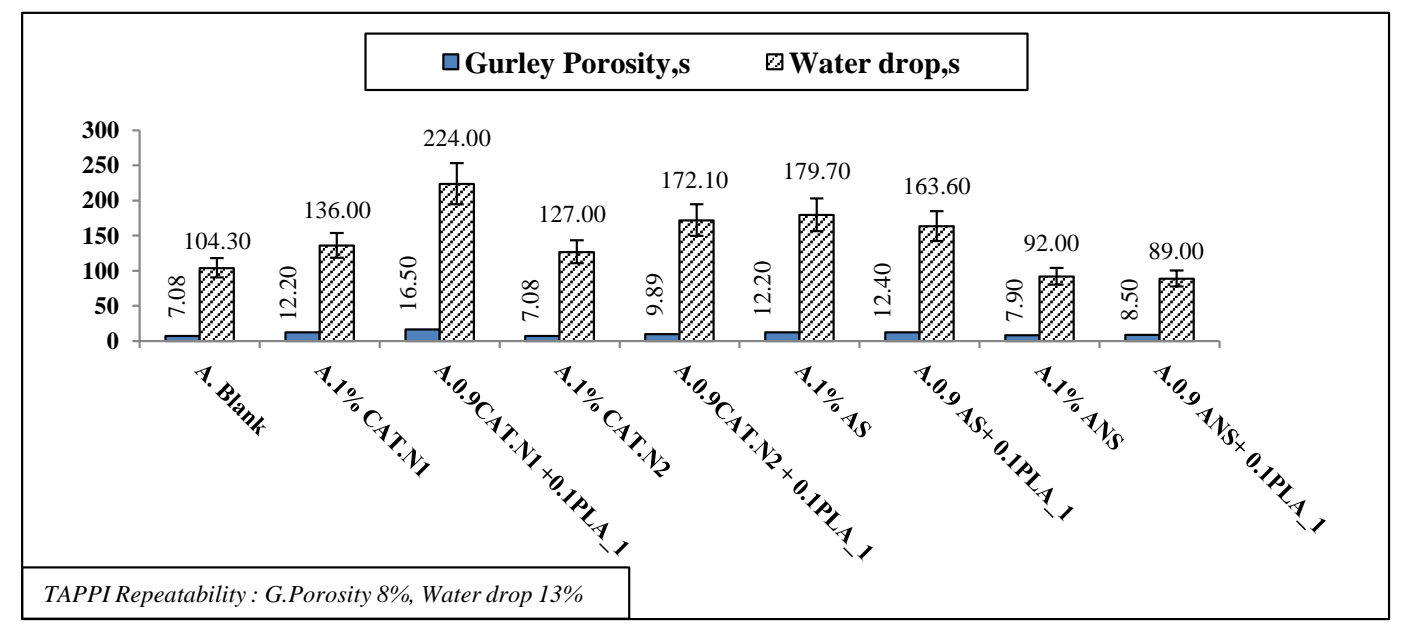

Figure 8. Effects of different starches on porosity and water drop of Pulp A.

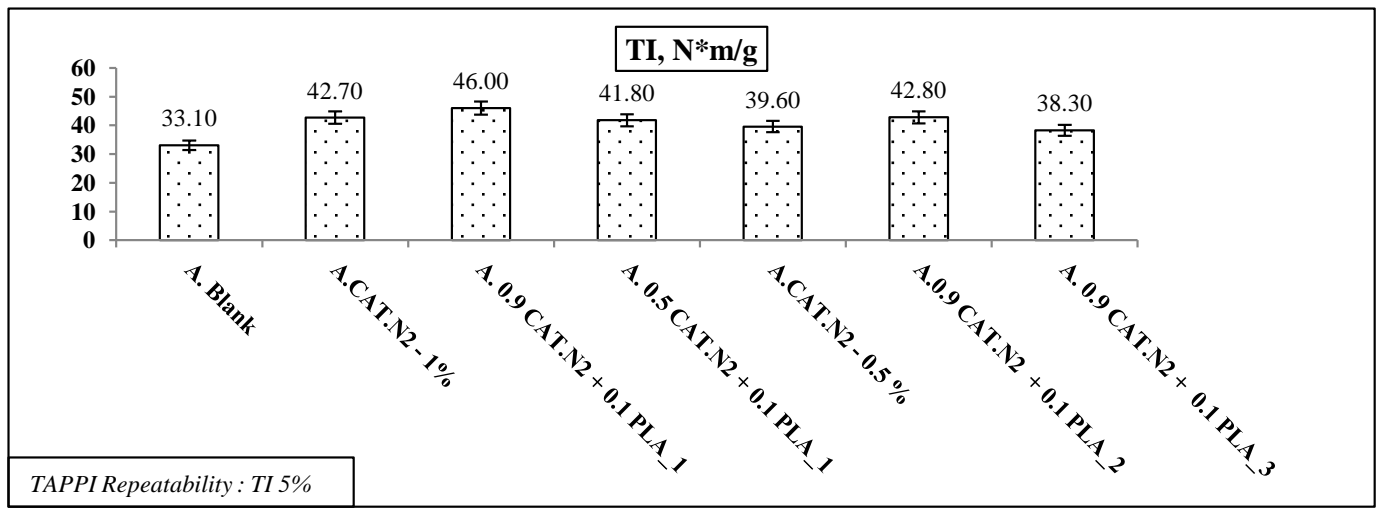

Figure 9. Effects of different starch ratios and starch-PLA on TI of handsheets.

It can be seen from Figure 9 that the highest TI was recorded for the combination (Pulp A + 0.9\% CAT.N2 + $0.1 \%$ PLA_1) at $46.00 \mathrm{Nm} / \mathrm{g}$. It is greater than the paper made from Pulp A plus 1\% CAT.N2 by an amount of $3.3 \mathrm{Nm} / \mathrm{g}$ ( $7.4 \%$ difference). The result suggests that by the substitution of $0.1 \%$ PLA_1, the bonding degree of fibers increases which leads to the increase in tensile strength of paper.

It is also clear that with the decrease in starch consumption, the TIs slightly decrease. Particularly, TI drops to $41.80 \mathrm{Nm} / \mathrm{g}$ and $39.60 \mathrm{Nm} / \mathrm{g}$ for (0.5\% CAT.N2 + 0.1\% PLA_1) and (0.5\% CAT.N2), respectively. The reason for the decrease in TIs is primarily due to the less bonding reinforcement between fibers since less starch is added to the furnish.

\subsection{Effects of Different PLAs on Paper Properties (Pulp A, CAT.N2)}

Figure 10 displays the effect of different PLA MWs on selected properties of paper handsheets made from pulp A and cationic starch CAT.N2.

For the TI, the use of $0.1 \%$ PLA_1 with $0.9 \%$ CAT.N2 brings paper handsheets the highest value of TI at $46.00 \mathrm{Nm} / \mathrm{g}$ while paper handsheets made with 0.1\% PLA_3 and 0.9\% CAT.N2 have the lowest TI at 38.30 $\mathrm{Nm} / \mathrm{g}$. Significantly, the increase of TI for the 1\% CAT.N2 compared to the blank is $9.6 \mathrm{Nm} / \mathrm{g}$ (42.70 vs. 33.10 $\mathrm{Nm} / \mathrm{g}$, about 29\% increase). Furthermore, it is clearly seen that TI for the 0.1\% PLA_1 with 0.9\% CAT.N2 $(46.00 \mathrm{Nm} / \mathrm{g})$ is higher about $5.9 \%$ than that of the 1\% CAT.N2 (42.70 Nm/g). Being lower than TI of paper made with PLA_1, the TI of paper made with 0.9\% starch + 0.1 PLA_2 is $42.80 \mathrm{Nm} / \mathrm{g}$ which is almost the same with TI of paper made with 1\% CAT.N2. The results suggest that PLA_1 and PLA_2 are potential wet end agents to use with starch for tensile strength reinforcement.

Moving to Wet TS, it is clear that wet TS has a very similar trend with TI discussed above. The combination 


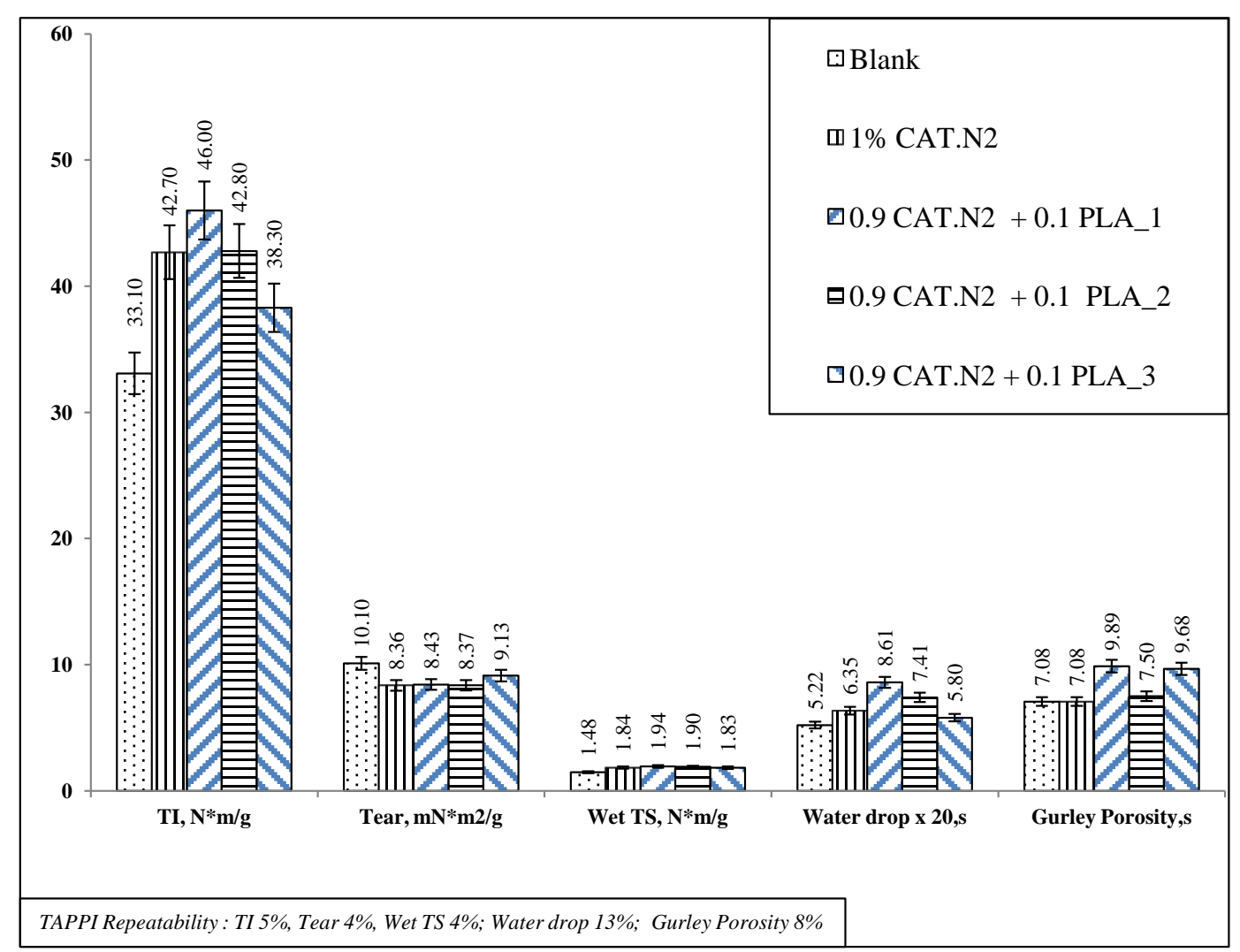

Figure 10. Effects of different PLAs on paper properties (Pulp A, CAT.N2).

of CAT.N2 and PLA_1 also gives the highest value of wet TS $(1.94 \mathrm{Nm} / \mathrm{g})$ while the lowest value is recorded for paper made with PLA_3 $(1.83 \mathrm{Nm} / \mathrm{g})$. Therefore, PLA_1 and PLA_2 are also potential agents for wet tensile strength reinforcement.

For tear result, it can be seen that the highest tear index belongs to the blank experiment. This is because the TI of the blank experiment is lowest, resulting in the increase of tear due to the bonding degree of fibers. Among the tear of paper made from the three PLAs, the use of PLA_3 gives the best value of tear at $9.13 \mathrm{mN} * \mathrm{~m}^{2} / \mathrm{g}$. Tear index values of paper made with PLA_1 and PLA_2 are quite similar and are lower than that of paper made with PLA_3.

In regards to water resistance, the longest time for a drop of water to completely penetrate through paper sample is belonged to paper made with PLA_1, at $8.61 \times 20 \mathrm{~s}(172.20 \mathrm{~s})$. The water drop value for paper made with PLA_2 is seen at $7.41 \times 20 \mathrm{~s}(148.20 \mathrm{~s})$, which is higher than the value of $1 \%$ CAT.N2 alone. The results also suggest that PLA_1 and PLA_2 are considered to be good agents for water resistance.

Gurley porosity values of paper made from all the three PLAs are clearly higher than that of paper made with no additive and with 1\% CAT.N2. Among of the three PLAs, paper handsheet made with PLA_1 and PLA_3 show similar value of Gurley porosity at 9.89 and 9.68 seconds while the lowest value is recorded for paper made with PLA_2 at 7.50 seconds. In general, paper handsheet made from Pulp A with the combination of PLA_1 + CAT.N2 gives the best response for dry tensile strength, wet TS, air and water resistance.

Figure 11 shows a plot of tear and tensile strength of paper handsheet made from Pulp A (high kappa number), with the use of high charge cationic CAT.N2, and the three different molecular weight of PLA. The linear (standard line) shows the normal expectation for tear and tensile relationship.

As can be seen from Figure 11, paper handsheet made from Pulp A + 0.9 CAT.N2 + 0.1PLA_1 shows the best response of strength, having the highest TI at $46 \mathrm{Nm} / \mathrm{g}$, but also having the tear value of $8.43 \mathrm{MN} * \mathrm{~m}^{2} / \mathrm{g}$. Handsheet from Pulp A + CAT.N2 1\% only, and handsheet from Pulp A + 0.9\% CAT.N2 + 0.1\% PLA_2 also have a good correlation of tear and tensile strength, which are seen very close to the line. The blank handsheet shows the highest tear index, but the tensile index is lowest, therefore it is not a good correlation. The results 


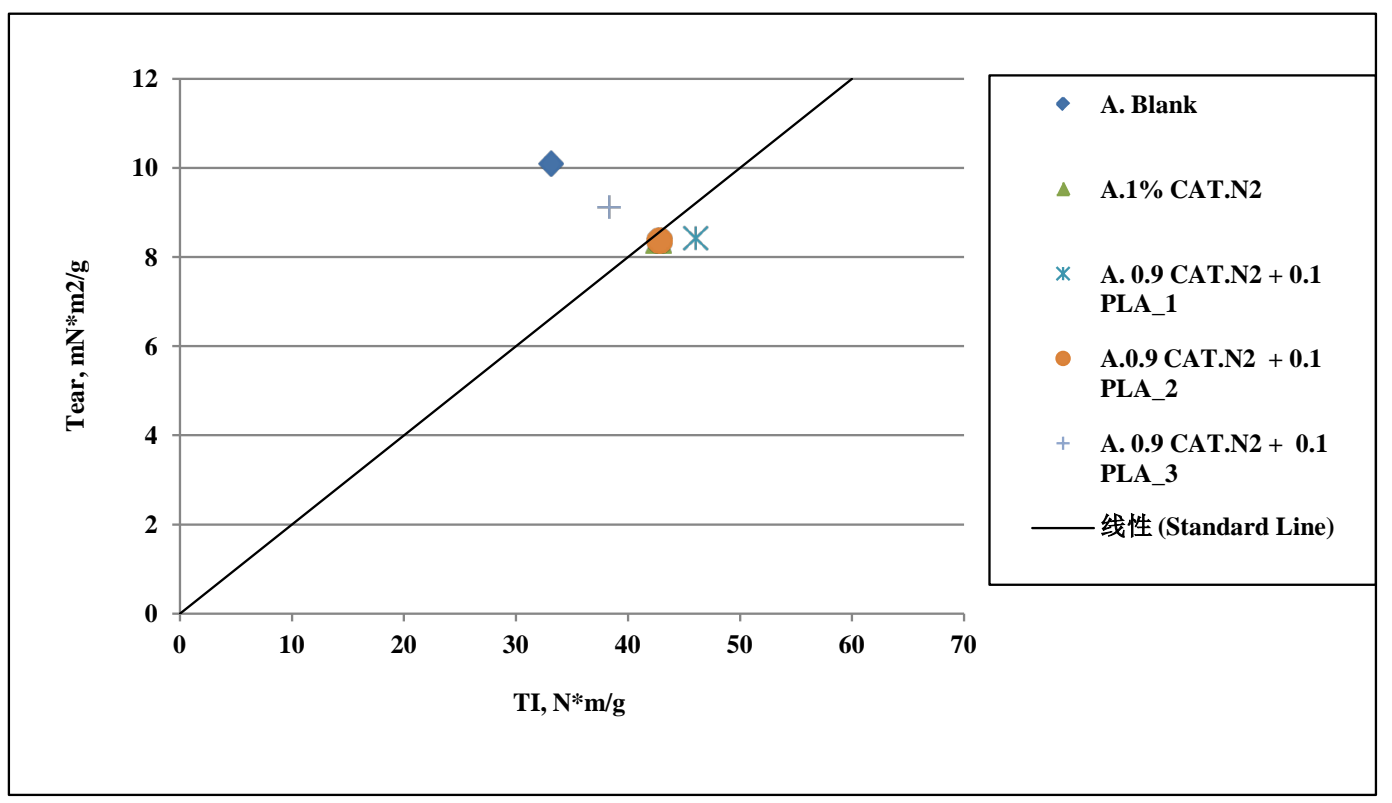

Figure 11. Plot of tear and tensile strength.

suggest that a papermaker will have different options to choose the optimal relationship between tear and tensile strength, depending on their products.

\subsection{Effects of Increasing the Amount of PLA_1 on Tensile Index for Pulp C}

Figure 12 shows the effect of different amounts of PLA_1 on TI of Pulp C. Pulp C is the same as Pulp A, but they are refined to different CSFs. Pulp A has CSF of $305 \mathrm{ml}$ while Pulp C has CSF of $298 \mathrm{ml}$.

It can be seen from Figure 12 that with the use of 0.1\% PLA_1 TI of paper made with amphoteric starch AS $(45.50 \mathrm{Nm} / \mathrm{g})$ is higher than that $(41.00 \mathrm{Nm} / \mathrm{g})$ of paper made with $1 \%$ AS. However, with the use of $0.2 \%$ of PLA_1, TI of paper made with AS significantly decreases back to $40.70 \mathrm{Nm} / \mathrm{g}$.

For the cationic starch CAT.N1, with the use of 0.1\% PLA_1 TI (40.40 Nm/g) is lower than that (44.10 Nm/g) of paper handsheets made with 1\% CAT.N1. When $0.2 \%$ of PLA_1 is used, TI increases slightly to $42.00 \mathrm{Nm} / \mathrm{g}$ but this increase is not significant due to being less than $5 \%$ repeatability.

\subsection{Effect of PLA Used as Surface Sizing Agent on TI and Cobb Value of Linerboard}

Figure13 expresses the effect of PLA on TI and Cobb value of linerboard when PLA is used as a surface sizing agent.

As can be seen TI of the paper treated with the highest MW 140,000 - 160,000 is recorded to be greatest at $54.00 \mathrm{Nm} / \mathrm{g}$. Comparing to the blank samples, there is a slight improvement in TI for samples treated with PLA_2 (53.00 vs. 50.30) and PLA_3 (54.00 vs. 50.30).

Linerboard treated with PLA_1 has a lower Cobb value $\left(22.10 \mathrm{~g}\right.$ of water/ $\left.\mathrm{m}^{2}\right)$ than the blank sample (23.00 g of water $/ \mathrm{m}^{2}$ ) which was not treated with PLA. That means PLA_1 has a minor effect in helping paper to resist water. On the other hand, interestingly Cobb values of paper treated with PLA_2 and PLA_3 are seen to have the higher amount of water in the paper at 28.60 and $26.20 \mathrm{~g}$ of water $/ \mathrm{m}^{2}$ than the blank at $23.00 \mathrm{~g}$ of water $/ \mathrm{m}^{2}$. That suggests that with the present of PLA_2 and PLA_3, the water resistance of paper decreased. One of the possible reasons for the decrease is that PLAs may react with an internal sizing agent AKD, resulting in the reduction in sizing efficiency of AKD for linerboard.

\subsection{Comparative Effect of the PLAs as Surface Sizing \& Wet End Agent on TI}

In this study, PLAs were used for two different purposes, as surface sizing agent and wet end agent. Figure 14 illustrates the different effects of PLAs on TI when PLAs plays the different roles. 


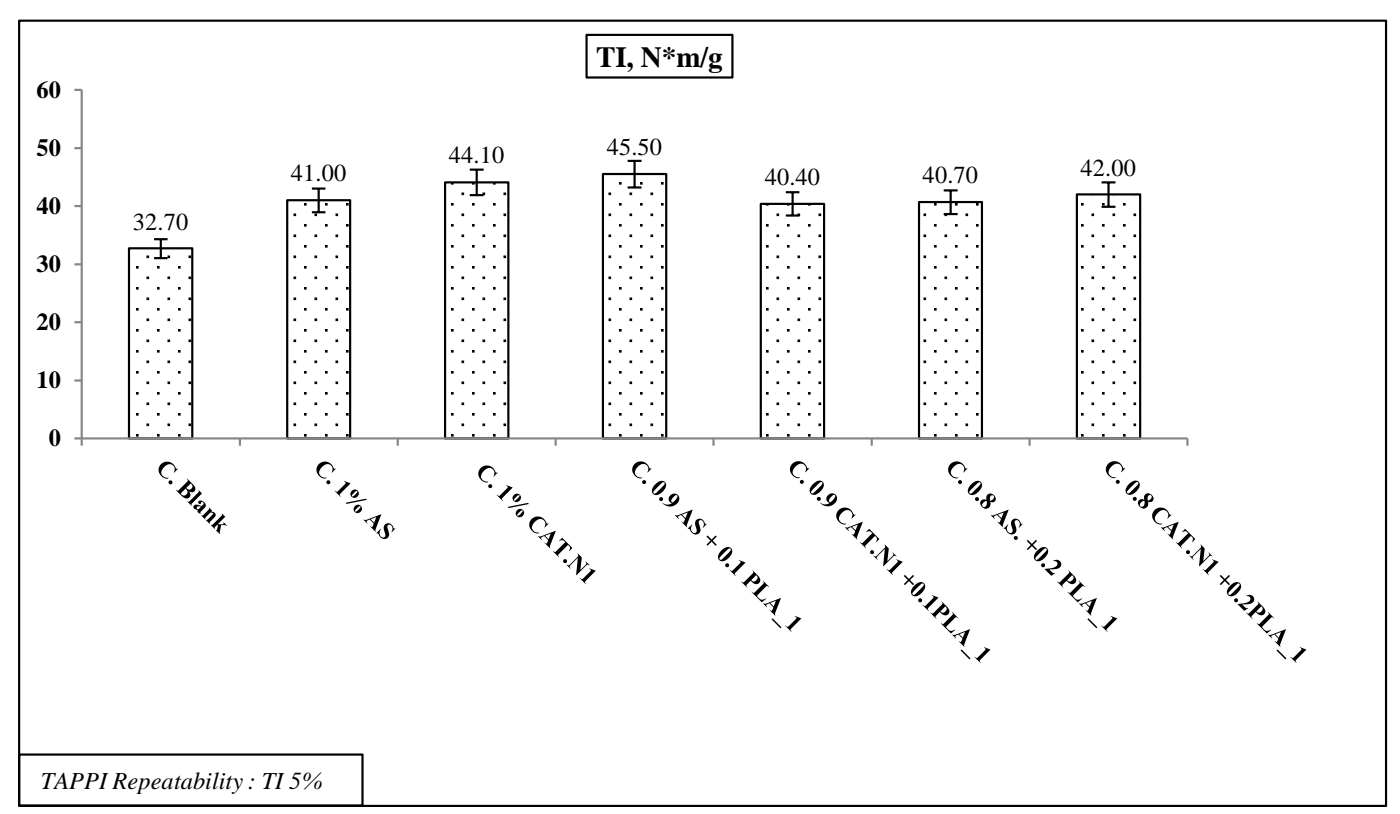

Figure 12. Effects of increasing the amount of PLA_1 on tensile index of Pulp C.

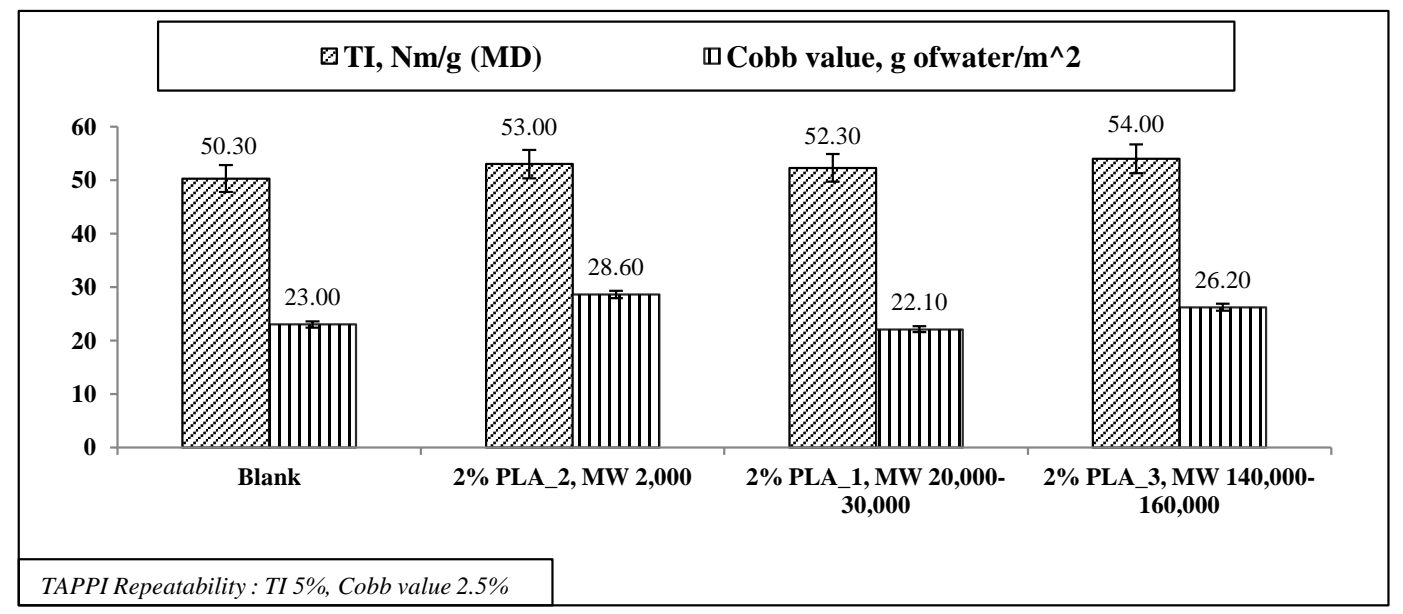

Figure 13. Effect on TI and Cobb value when PLA is used as a surface sizing agent.

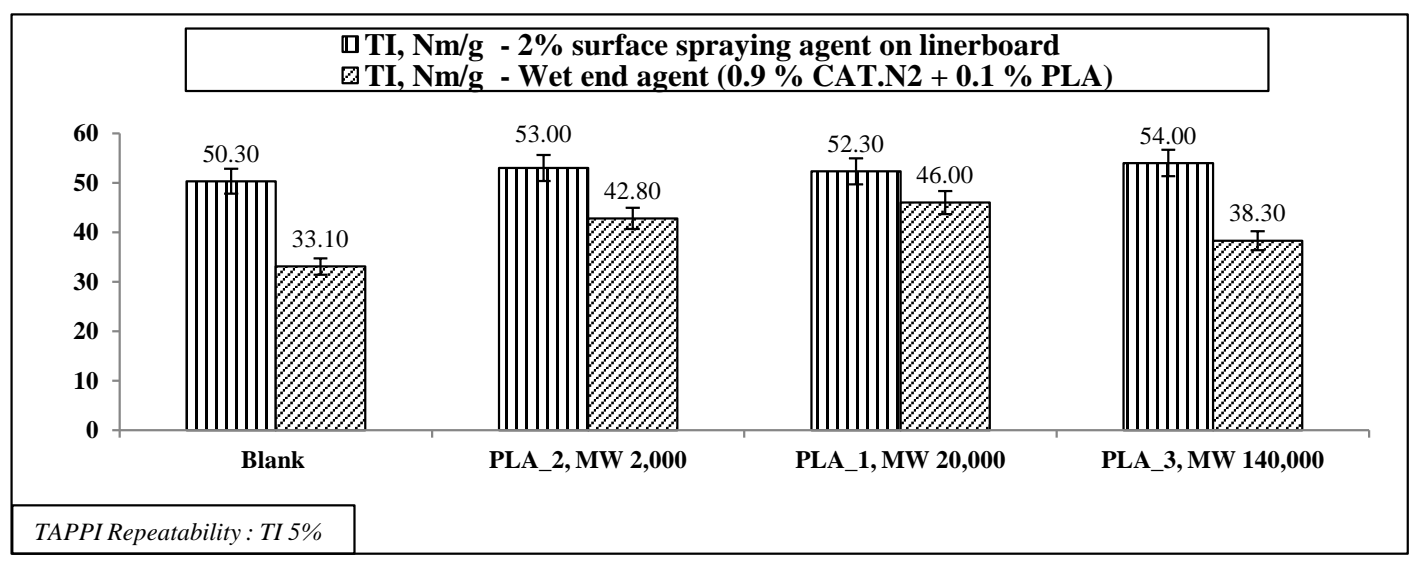

Figure 14. Effect of different PLAs to TI as PLAs are a surface sizing and a wet end agent. 
As wet end agent, the graph shows that PLA_1 with MW of 20,000 gives the greatest result in TI at 46.00 $\mathrm{Nm} / \mathrm{g}$. Interestingly, the TI $(42.80 \mathrm{Nm} / \mathrm{g})$ of paper made from PLA_2 with lowest MW is higher than that (38.30 Nm/g) of paper made from PLA_3 with highest MW $(140,000)$.

As a surface sizing agent on linerboard, the graph shows slight increase in TIs for all the three different PLAs compared to TI of blank which was not treated with PLA. However, it can be seen that there is no significant difference in TI when comparing the three PLAs with each other. In other words, the difference in TI of each pair is less than $5 \%$ repeatability. Therefore, it can be concluded that PLAs are not important in the case of a surface sizing agent for linerboard.

\section{Conclusions}

Our results indicate that the effect of PLAs on the selected properties including strength of paper handsheets made from the three different pulps depends on the type of starch, on the molecular weight of PLA, and on the lignin content in the pulps.

1) The paper handsheets made from higher kappa number pulp (Pulp A), combining with the medium molecular weight PLA at $0.1 \%$ (PLA_1, 20,000 - 30,000), and with higher charge cationic starch at $0.9 \%$ (CAT.N2, \%N $=0.43$ ) gives the highest improvement for tensile strength, wet tensile strength, air and water resistance. This result verifies that higher kappa number pulp has better attraction to the hydrophobic PLA. Moreover, the results also show that the higher charge cationic starch is retained more on fibers and associated more with negative surface charge of fibers.

2) For paper handsheets made from the higher kappa number pulp, with the use of 0.1\% PLA_1 the values of Zeta Potential and $\mathrm{pH}$ show that the white water suspension is stable and is similar to the values in the condition that no PLA_1 is used. Another conclusion is that the use of 0.1\% PLA_1 + 0.9\% CAT.N1 is better than the use of $0.2 \%$ PLA_1 + 0.8\% CAT.N1 for tensile strength.

3) When PLAs are used as surface sizing agents for linerboard, the improvement in tensile strength for paper treated with PLA_2 (MW 2000) and PLA_3 (MW 140,000) is higher than that of paper treated with PLA_1. On the other hand, when PLAs are used as wet end agents, the PLA_1 gives better response than PLA_2 and PLA_3 in terms of tensile strength.

4) The result of this study interestingly shows that when lignin-rich pulp is employed, tensile strength of paper handsheet made with the combination of amphoteric starch + PLA_1 is very comparable with that of paper handsheet made with high cationic charge starch + PLA_1. This result verifies that, amphoteric starch is a very promising substitution for high cationic charge starch when combining with the medium molecule weight PLA to improve tensile strength of paper.

\section{Acknowledgements}

The authors are grateful for the support of the Department of Paper and Bioprocess Engineering at the State University of New York, College of Environmental Science and Forestry.

\section{References}

[1] Scott, W.E. (1996) Principles of Wet End Chemistry. TAPPI Press.

[2] Biermann, C.J. (1996) Handbook of Pulping and Papermaking. 2nd Edition, Academic Press Limited, London.

[3] Neimo, L. (1999) Papermaking Science and Technology: Papermaking Chemistry. Fapet Oy, Helsinki, $268-301$.

[4] Gong, C., Hasan, A., Bujanovic, B.M. and Amidon, T.E. (2012) Novel Blend of Biorenewable Wet-End Paper Agents. APPI, 11, 41-48.

[5] Hasan, A., Bujanovic, B.M. and Amidon, T.E. (2010) Strength Properties of Kraft Pulp Produced from Hot-Water Extracted Wood Chips within the Biorefinery. Journal of Biobased Materials and Bioenergy, 4, 46-52. http://dx.doi.org/10.1166/jbmb.2010.1064

[6] Smook, G.A. (2002) Handbook for Pulp and Paper Technologists. 3rd Edition, Angus Wilde Publications, Inc., 207. 


\section{TAPPI Standards}

T236 om-06: “Kappa number of pulp”.

T227 om-09: "Freeness of pulp (Canadian standard method)”.

T205 sp-06: "Forming handsheets for physical tests of pulp".

T220 sp-10: "Physical testing of pulp handsheets".

T494 om-06: "Tensile properties of paper and paperboard (using constant rate of elongation apparatus)”.

T456 om-10: "Tensile breaking strength of water-saturated paper and paperboard ('wet tensile strength')”.

T460 om-11: “Air resistance of paper (Gurley method)”.

T414 om-12: "Internal tearing resistance of paper (Elmendorf-type method)".

T441 om-09: "Water absorptiveness of sized (non-bibulous) paper, paperboard, and corrugated fiberboard (Cobb test)”.

T 835 om-08: "Water absorption of corrugating medium: water drop absorption test”. 
Scientific Research Publishing (SCIRP) is one of the largest Open Access journal publishers. It is currently publishing more than 200 open access, online, peer-reviewed journals covering a wide range of academic disciplines. SCIRP serves the worldwide academic communities and contributes to the progress and application of science with its publication.

Other selected journals from SCIRP are listed as below. Submit your manuscript to us via either submit@scirp.org or Online Submission Portal.
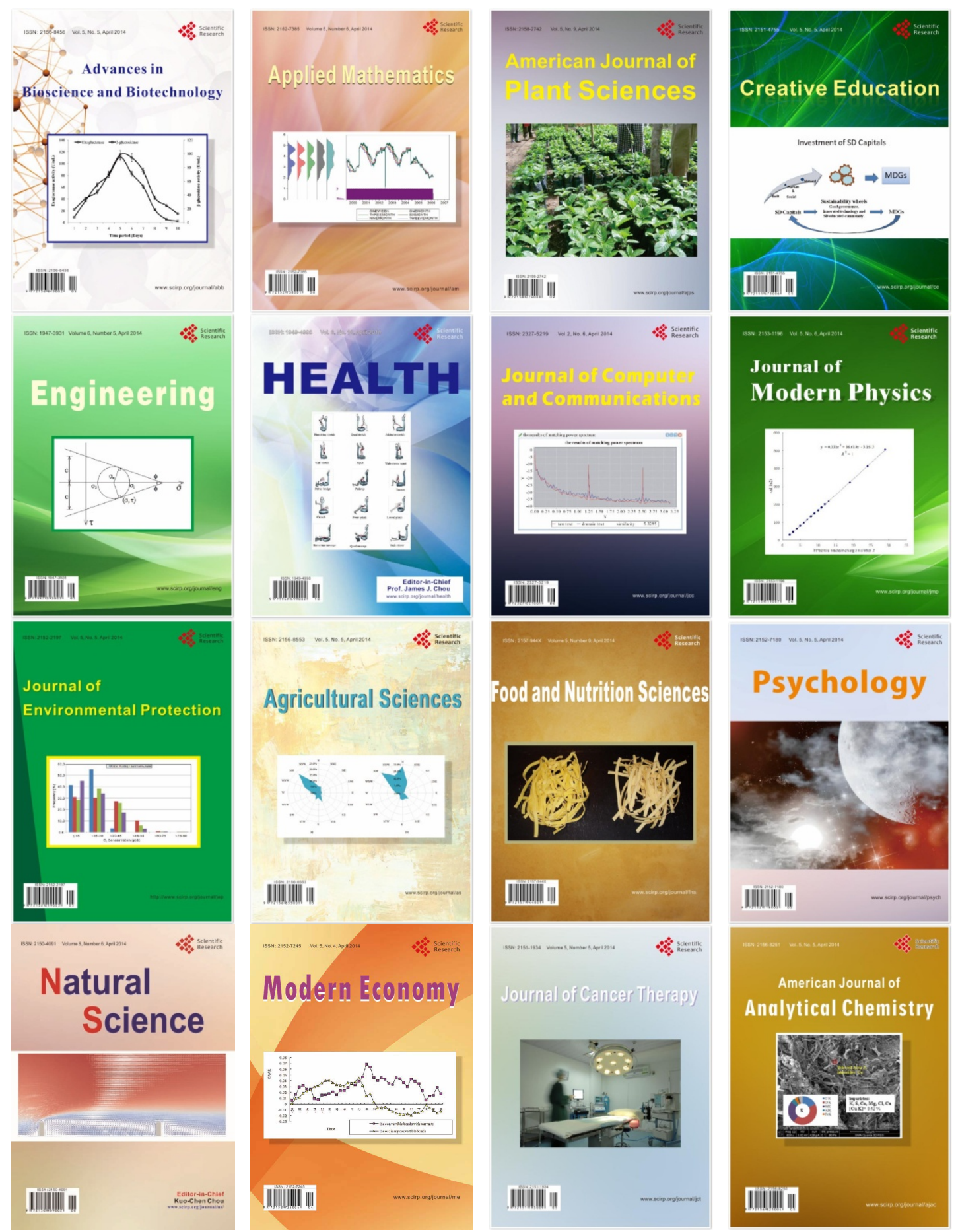